\title{
Neutrino masses from large extra dimensions
}

\author{
Nima Arkani-Hamed \\ SLAC, Stanford University, Stanford, California 94309 \\ Savas Dimopoulos \\ Physics Department, Stanford University, Stanford, California 94305 \\ Gia Dvali \\ Physics Department, New York University, New York, New York 10003 \\ and ICTP, Trieste, Italy \\ John March-Russell \\ Theory Division, CERN, CH-1211, Geneva 23, Switzerland \\ (Received 9 February 1999; published 26 December 2001)
}

\begin{abstract}
Recently it was proposed that the standard model (SM) degrees of freedom reside on a $(3+1)$-dimensional wall or "3-brane" embedded in a higher-dimensional spacetime. Furthermore, in this picture it is possible for the fundamental Planck mass $M_{*}$ to be as small as the weak scale $M_{*} \simeq O(\mathrm{TeV})$ and the observed weakness of gravity at long distances is due the existence of new submillimeter spatial dimensions. We show that in this picture it is natural to expect neutrino masses to occur in the $10^{-1}-10^{-4} \mathrm{eV}$ range, despite the lack of any fundamental scale higher than $M_{*}$. Such suppressed neutrino masses are not the result of a seesaw, but have intrinsically higher-dimensional explanations. We explore two possibilities. The first mechanism identifies any massless bulk fermions as right-handed neutrinos. These give naturally small Dirac masses for the same reason that gravity is weak at long distances in this framework. The second mechanism takes advantage of the large infrared desert: the space in the extra dimensions. Here, small Majorana neutrino masses are generated by a breaking lepton number on distant branes.
\end{abstract}

DOI: 10.1103/PhysRevD.65.024032

PACS number(s): 04.50.+h, 14.60.Pq

\section{INTRODUCTION}

It has recently become clear that the fundamental scale of gravity need not be the Planck scale $M_{\mathrm{pl}} \simeq 1.2 \times 10^{19} \mathrm{GeV}$, but rather that the true scale $M_{*}$ where gravity becomes strong can be much lower. The observed small value of Newton's constant at long distances is ascribed to the spreading of the gravitational force in $n$ "large" extra dimensions. The volume $R^{n}$ of the new dimensions is fixed by Gauss's law to be

$$
R^{n} \simeq M_{\mathrm{pl}}^{2} / M_{*}^{n+2} .
$$

The most radical, and in many ways the most attractive, suggestion for $M_{*}$ is that it should be close to the weak scale $M_{*} \sim 1 \mathrm{TeV}$. In this case we have $R \simeq 10^{-17+30 / n} \mathrm{~cm}$. For $n=1, R \sim 10^{13} \mathrm{~cm}$, so this case is excluded since it would modify Newtonian gravitation at solar-system distances. Already for $n=2$, however, $R \sim 1 \mathrm{~mm}$, which happens to be the distance where our present experimental knowledge of gravitational strength forces ends.

While the gravitational force has not been measured beneath a millimeter, the success of the Srandard Model (SM) up to $\sim 100 \mathrm{GeV}$ implies that the SM fields cannot feel the extra large dimensions; that is, they must be stuck on a 3-dimensional wall, or "3-brane," in the higher-dimensional space. Thus, in this framework the universe is $(4+n)$ dimensional with the fundamental Planck scale $M_{*}$ residing somewhere between the weak scale and $M_{\mathrm{pl}}$, with new submillimeter-sized dimensions where gravity, and perhaps other fields, can freely propagate, but where the SM particles are localized on a 3-brane in the higher-dimensional space [1-4].

The most attractive possibility for localizing the SM fields to the brane is to employ the D-branes that naturally occur in type I or type II string theory [5,3]. Gauge and other degrees of freedom are naturally confined to such D-branes [5] and, furthermore, this approach has the advantage of being formulated within a consistent theory of gravity. However, from a practical point of view, the most important question is whether this framework is experimentally excluded. This was the subject of [4] where laboratory, astrophysical, and cosmological constraints were studied and found not to exclude these ideas, even for $M_{*}$ as low as $1 \mathrm{TeV}$. There are a number of model independent predictions of such a scenario, ranging from the production of Regge excitations and bulk gravitons at the next generation of colliders $[2,3,6]$, to the modification of the properties of black holes [7].

There are also a number of other papers discussing related suggestions. Reference [8] examine the idea of lowering the grand unified theory (GUT) scale by utilizing higher dimensions. Further papers concern themselves with the construction of string models with extra dimensions larger than the string scale [9-11], and gauge coupling unification in higher dimensions without lowering the unification scale [12]. There are also important papers by Sundrum on the effective theory of the low energy degrees of freedom in realizations of our world as a brane, and on radius stabilization $[13,14]$. For earlier works on the world as a three-dimensional wall, 
see [15]. The issue of radius stabilization was also considered in $[16]$.

However, it may seem that we have given up any hope of explaining the size of the neutrino masses deduced to be necessary to explain the atmospheric [17] and solar [18] neutrino anomalies. In the traditional approach the small neutrino masses are the result of the seesaw mechanism, in which a large right-handed (RH) Majorana mass $M_{R}$ suppresses one of the eigenvalues of the neutrino mass matrix, leading to $m_{\nu} \sim m_{\text {fermion }}^{2} / M_{R}$. The neutrino mixing explanations of the atmospheric and solar neutrino anomalies require $M_{R}$ to be a superheavy mass scale, varying between an intermediate scale $\sim 10^{10} \mathrm{GeV}$ the GUT scale. However, in the world-as-a-brane picture with $M_{*} \sim 1 \mathrm{TeV}$ the existence of such a superheavy scale is unpalatable.

In this paper we show that there are intrinsically higherdimensional explanations for either Dirac or Majorana neutrino masses. For Dirac masses, the basic idea is that any fermionic state that propagates in the bulk must, by definition, be a SM singlet and, furthermore, that it couples to the wall-localized SM states precisely as a right-handed neutrino with a naturally small coupling. The small coupling is a result of the large relative volume of the internal "bulk" manifold compared to the thin wall where SM states propagate. The interaction probability of the Kaluza-Klein (KK) zero mode of the bulk RH neutrino state $\nu_{R}$ with the branelocalized Higgs and lepton doublet fields is thus small, resulting in a greatly suppressed $\nu_{R}(x, y=0) L(x) H(x)$ coupling. Small Majorana masses can be obtained using the generic mechanism of [19] for generating small couplings by breaking symmetries on distant branes in the bulk. In our context, we break the lepton number on far-away branes, and have this breaking communicated to us by bulk messenger fields, giving a naturally distance-suppressed Majorana neutrino mass on our wall.

\section{RIGHT-HANDED NEUTRINOS IN THE BULK}

In this section, we will show that neutrinos can acquire naturally small Dirac masses if the left-handed neutrinos on our wall couple to any massless bulk fermion. Since the SM gauge fields are localized on our 3-brane, a bulk fermion must be a SM singlet, and will henceforth be referred to as the bulk right-handed neutrino in this section. The reason for the suppressed mass is that bulk modes have couplings suppressed by the volume of the extra dimensions; this is the reason for the weakness of gravity at long distances in our scenario, as well as small gauge couplings for bulk gauge fields $[4,19,20]$.

For simplicity, we begin by considering a toy 5-dimensional theory to concretely illustrate the idea; the generalization to the physically realistic case of higher dimensions will then be clear. Consider a 5-dimensional theory with coordinates $\left(x^{\mu}, y\right)$, with $\mu=0, \ldots, 3$ and the $y$ direction compactified on a circle of circumference $2 \pi R$ by making the periodic identification $y \sim y+2 \pi R$. Our 3-brane, where the lepton doublet and the Higgs fields are localized, is located at $y=0$, while a massless Dirac fermion $\Psi$ propa- gates in the full five dimensions. The $\Gamma$ matrices can be written as

$$
\Gamma^{\mu}=\left(\begin{array}{cc}
0 & \sigma^{\mu} \\
\bar{\sigma}^{\mu} & 0
\end{array}\right), \quad \Gamma^{5}=\left(\begin{array}{cc}
i & 0 \\
0 & -i
\end{array}\right)
$$

where we have chosen the Weyl basis for the $\Gamma^{\mu}$ matrices. The Dirac spinor $\Psi$ is also conveniently decomposed as usual in the Weyl basis

$$
\Psi=\left(\begin{array}{c}
\nu_{R} \\
{\overline{\nu^{c}}}_{R}^{c}
\end{array}\right) .
$$

Let us now shut off all interactions between bulk and wall fields and understand the spectrum of the theory from the 4-dimensional point of view. If we Fourier expand

$$
\nu_{R}^{(c)}(x, y)=\sum_{n} \frac{1}{\sqrt{2 \pi R}} \nu_{R n}^{(c)}(x) e^{i n y / R}
$$

then the free action for $\Psi$ becomes

$$
S_{\Psi}^{\mathrm{free}}=\int d^{4} x \sum_{n} \bar{\nu}_{R n} \bar{\sigma}^{\mu} \nu_{R n}+{\overline{\nu^{c}}}_{R n} \bar{\sigma}^{\mu} \nu_{R n}^{c}+\frac{n}{R} \nu_{R n} \nu_{R n}^{c}+\text { H.c. }
$$

Of course this is the usual Kaluza-Klein (KK) expansion, with the expected result. We have a tower of fermions $\nu_{R n}, \nu_{R n}^{c}$ with Dirac masses $n / R$ quantized in units of $1 / R$. The free action for the Lepton doublet $l$ localized on the wall is just

$$
S_{l}^{\mathrm{free}}=\int d^{4} x \bar{l} \bar{\sigma}^{\mu} l .
$$

Let us now imagine writing down the most general interactions between wall and bulk fields. Since something analogous to the lepton number must be imposed to forbid toolarge Majorana neutrino masses for the SM fields anyway, we will for simplicity assume that the lepton number is conserved and assign $\Psi$ the opposite lepton number of $L$. The leading local interaction between $\Psi$ and wall fields is then

$$
S^{\mathrm{int}}=\int d^{4} x \kappa l(x) h^{*}(x) \nu_{R}(x, y=0)
$$

where $\kappa$ is a dimensionless coefficient and we work in units where the fundamental scale $M_{*}=1$. Notice that this coupling manifestly breaks the full 5-dimensional Poincare invariance of the theory by picking out the component $\nu_{R}$ from the full Dirac spinor $\Psi$. This is perfectly reasonable, since the presence of the wall itself breaks the 5-dimensional Poincaré invariance to the 4-dimensional one, and therefore the couplings need only be invariant under the 4-d Poincaré transformations. As we show in the Appendix, this can be seen very explicitly in a specific setup for localizing $l, H$ on a $(3+1)$-dimensional domain wall in $4+1$ dimensions. Upon setting the Higgs field to its vacuum expectation value 
VEV $v$, and expanding $\psi(x, y=0)$ in KK modes, the above interaction generates the following mass terms:

$$
S^{\mathrm{int}}=\int d^{4} x \frac{\kappa v}{\sqrt{2 \pi R}} \nu_{L}(x) \sum_{n} \nu_{R n}(x)
$$

Suppose that $\kappa v / \sqrt{R} \ll 1 / R$. In this case, all the massive KK excitations are unaffected by this term. However, this interaction generates a Dirac mass term between $\nu_{L}$ and the zero mode $\nu_{R 0}$, which is suppressed by the size of the dimensions:

$$
m_{\nu}=\kappa \frac{v}{\sqrt{R}}
$$

It is clear that this generalizes to the case where the righthanded neutrino lives in any number $n$ of extra dimensions. In the decomposition of a higher-dimensional spinor under the 4D Lorentz group, there will be a number of left-handed Weyl spinors which can have an interaction of the type in Eq. (7), which gives a mass term suppressed by (volume) $)^{-1 / 2}$ between $\nu_{L}$ and all the KK excitations of the bulk righthanded neutrino. As long as this mass is smaller than $1 / R$, this is negligible for the KK modes but gives a Dirac mass

$$
m_{\nu}=\kappa \frac{v}{\sqrt{V_{n} M_{*}^{n}}}
$$

where we have restored the $M_{*}$ dependence. Upon using the relation $M_{p l}^{2}=M_{*}^{n+2} V_{n}$, we obtain for the neutrino mass

$$
m_{\nu}=\kappa \frac{v M_{*}}{M_{p l}} \sim 10^{-4} \mathrm{eV} \frac{\kappa M_{*}}{1 \mathrm{TeV}}
$$

Note that for all $n>2$, this mass is much smaller than $1 / R$ so our analysis was justified, while for $n=2$ they are roughly comparable; this will pose phenomenological difficulties for $n=2$ as discussed in Sec. V, and henceforth we shall only consider cases with $n>2$. It is remarkable that for the case of a low string scale $\kappa M_{*} \sim 1-100 \mathrm{TeV}$, this prediction for the neutrino masses is very roughly in the right range to explain the atmospheric and solar neutrino anomalies.

Let us more carefully compute the neutrino mass, by integrating out the $\mathrm{KK}$ modes. Integrating out the massive $\psi^{(\vec{n})}, \psi^{c(\vec{n})}$ pair at tree level generates the operator

$$
\frac{1}{|\vec{n}|^{2} / R^{2}} \bar{l} \bar{\sigma}^{\mu} \partial_{\mu} l h^{*} h .
$$

The sum over all KK modes is power divergent in the UV for $n>2$. This UV divergence must be cutoff near the fundamental scale $M_{*}$, i.e. at a $|k|_{\text {max }}$ such that $\left|k_{\text {max }}\right| / R$ $=c M_{*}$, where $c$ is a dimensionless factor reflecting our ignorance of where exactly this power divergence is cutoff. The generated operator is

$$
\frac{\kappa^{2} c^{n-2}}{M_{*}^{2}} \bar{l} \bar{\sigma}^{\mu} \partial_{\mu} l h^{*} h
$$

After setting the Higgs field to its VEV, this generates a correction to the $\nu_{L}$ wave function renormalization. After going back to canonical normalization for $\nu_{L}$, the neutrino mass becomes

$$
m_{\nu}=\frac{\kappa}{\sqrt{1+\kappa^{2} c^{n-2} v^{2} / M_{*}^{2}}} \frac{v M_{*}}{M_{p l}} .
$$

The significance of this equation is that for a fixed value of $M_{*}$ it is not possible to increase the neutrino mass arbitrarily by increasing $\kappa$, rather there is an upper bound

$$
m_{\nu}^{\max }=c^{-(n-2) / 2} M_{*}^{2} / M_{p l}
$$

All of this can be seen more explicitly by simply writing down the mass matrix for the various neutrino fields; for simplicity let us consider the case $n=1$. The relevant fields with $L=1$ are $N_{+}=\left(\nu_{L}, \nu_{R 1}^{c}, \nu_{R 2}^{c}, \ldots\right)$, while those with $L=-1$ are $N_{-}=\left(\nu_{R 0}, \nu_{R 1}, \nu_{R 2}, \ldots\right)$. Note that $\nu_{R 0}^{c}$ does not acquire a mass term with any other field and remains exactly massless. The mass matrix is of the form

$$
\mathcal{L}_{\text {mass }}=N_{-}^{T} \mathcal{M} N_{+},
$$

with

$$
\mathcal{M}=\left(\begin{array}{cccc}
m & 0 & 0 & \ldots \\
m & 1 / R & 0 & \ldots \\
m & 0 & 2 / R & \ldots \\
\vdots & \vdots & \vdots & \ddots
\end{array}\right)
$$

where $m$ is as in Eq. (9). If we treat all the off-diagonal terms as perturbations, then at zeroth order the lightest eigenvalue of this matrix is $m$. To first order in perturbation theory, the eigenvalues are unchanged, but we find that the lightest $L$ $=1$ mass eigenstate is dominantly $\nu_{L}$, with an admixture of

$$
\theta_{n} \sim \frac{m}{|n| / R}
$$

of the KK mode $\nu_{R n}^{c}$. The first shift in the eigenvalues occurs at second order in perturbation theory. It is more convenient to use the Hermitian matrix $\mathcal{M} \mathcal{M}^{\dagger}$, whose eigenvalues are the absolute value squared of the eigenvalues of $M$ :

$$
\begin{aligned}
\mathcal{M} \mathcal{M}^{\dagger}= & \operatorname{diag}\left(m^{2},(1 / R)^{2},(2 / R)^{2}, \ldots\right) \\
& +\left(\begin{array}{cccc}
0 & 1 & 1 & \ldots \\
1 & 1 & 1 & \ldots \\
1 & 1 & 1 & \ldots \\
\vdots & \vdots & \vdots & \ddots
\end{array}\right) m^{2}
\end{aligned}
$$

The lowest eigenvalue gets corrected to be 


$$
m_{\nu}^{2} \rightarrow m_{\nu}^{2}\left(1-\sum_{n} \frac{m_{\nu}^{2} R^{2}}{n^{2}}\right) .
$$

Taking the square root, this is nothing but the first term in the perturbative expansion of Eq. (14).

\section{Right-handed neutrinos from sub-spaces}

The bulk fermion fields that give rise to the right-handed neutrinos on our brane do not necessarily live in the entire transverse $n$-dimensional bulk. It is consistent to suppose that they propagate in just an $m$-dimensional subspace $(m<n)$ of the entire bulk where gravity propagates. Such a situation can easily arise if our three-dimensional world is at the intersection point of two or more branes with at least one having $p=m+3>3$ spatial dimensions. Independent of how such a scenario is realized, the properties of the right-handed neutrino interactions with our wall localized states are simply described as a simple extension of the discussion in the previous section, which we do in a slightly different way below. Denote by $V_{m}$ the $m$-dimensional transverse volume in which the right-handed neutrino field propagates. Then once again the KK mode expansion of this field is

$$
\nu_{R}(x, y)=\frac{1}{\sqrt{V_{m}}} \sum_{\grave{\ell}} \nu_{R, \vec{\ell}}(x) \exp \left[-2 \pi i \vec{\ell} \cdot \vec{y} /\left(V_{m}\right)^{1 / m}\right] .
$$

The interaction of the KK zero mode $\vec{\ell}=0$ with an operator $\mathcal{O}$ constructed out of wall-localized standard model states is still given by the overlap integral

$$
\operatorname{Prob}=\int d^{3} x d^{n} y \mathcal{O}_{\mathrm{SM}}(x) \nu_{R, 0}(x, y) .
$$

Each standard model field in $\mathcal{O}$ has in its wave function a factor of $1 / \sqrt{V_{\text {wall }}}$ arising from the small wall extent in the $m$ transverse dimensions. Furthermore, there is a factor of $1 / \sqrt{V_{m}}$ from the normalization of the right-handed neutrino state, and a factor of $V_{\text {wall }} \sim 1 / M_{*}^{m}$ coming from the $\int d^{n} y$ integral which is only nonzero in the $m$-dimensional subspace where both the wall extends and the right-handed field propagates. Putting this together in the case of interest, the interaction term $\nu_{R} L H$ is suppressed by the probability

$$
\text { Prob }=\left(\frac{V_{\text {wall }}}{V_{m}}\right)^{1 / 2} .
$$

In the case of a symmetric internal manifold where each of the $n$ dimensions is of size $R$, the volume of the $m$-dimensional subspace is $V_{m} \sim R^{m}$. Thus upon using $M_{\mathrm{pl}}^{2}$ $=R^{n} M_{*}^{n+2}$ the factor in Eq. (23) reduces to

$$
\operatorname{Prob}=\left(\frac{M_{*}}{M_{\mathrm{pl}}}\right)^{m / n} \text {. }
$$

Including the power divergence of the normalization of the $\nu_{L}$ kinetic term, Eq. (13), adapted for the case where the right-handed neutrino propagates in $m<n$ dimensions, we have (for all the large dimensions of roughly equal size) the neutrino mass expression

$$
m_{\nu}=\frac{\kappa v}{\sqrt{1+c^{m-2} v^{2} / M_{*}^{2}}}\left(\frac{M_{*}}{M_{\mathrm{pl}}}\right)^{m / n} .
$$

Thus a large spectrum of neutrino masses is possible. For instance, if $n=6$ and $m=5$, even for $\kappa \sim 1$ and $M_{*}$ $\sim 1 \mathrm{TeV}$, we get $m_{\nu} \sim 10^{-2} \mathrm{eV}$, naturally the correct order of magnitude for explanations of the atmospheric neutrino anomaly.

In general we should note that there is no reason for the internal $n$-dimensional manifold to be symmetric. For instance in the case $n=6$ we could imagine compactifying on a product of two-tori $T^{2} \times T^{2} \times T^{2}$, each with its own characteristic radius. The Gauss's law condition for $M_{\mathrm{pl}}$ only requires that the total volume $V_{m}=M_{\mathrm{pl}}^{2} / M_{*}^{n+2}$. If we now define an average radius $R$ by the relation $R^{n}=V_{n}$, and write $V_{m}=V_{n} / V_{n-m}=R^{n} / V_{n-m}$, we get the general form of the suppression for the coupling $\nu_{R} L H$;

$$
\operatorname{Prob}=\left(\frac{M_{*}}{M_{\mathrm{pl}}}\right)^{m / n}\left(\frac{V_{n-m}}{R^{(n-m)}}\right)^{1 / 2} .
$$

\section{BREAKING THE LEPTON NUMBER ON DISTANT WALLS}

In the preceding sections, we have considered ways of obtaining naturally small Dirac masses for the neutrinos, in theories with a conserved lepton number. It is also possible to generate small Majorana neutrino masses, by using the generic idea of [19] for generating small couplings by breaking symmetries on distant branes. In our case, we wish to imagine that the lepton number is primordially good on our brane, but is maximally badly broken at the scale $M_{*}$ by the vev of a field $\varphi_{L}$ with the lepton number $L=2$ on a different brane located at $y=y_{*}$ in the extra dimensions. The information of this breaking is transmitted to us by a bulk field $\chi_{L}$ also carrying $L=2$. Working in units with $M_{*}=1$, the relevant interactions are

$$
\begin{gathered}
\mathcal{L} \supset \int_{\text {us }} d^{4} x \kappa\left(l h^{*}\right)^{2}(x) \chi_{L}(x, y=0) \\
\quad+\int_{\text {other }} d^{4} x^{\prime}\left\langle\varphi_{L}\right\rangle \chi_{L}\left(x, y=y_{*}\right) .
\end{gathered}
$$

The VEV of $\varphi_{L}$ on the other brane acts as a source for $\chi_{L}$, and "shines" $\chi_{L}$ everywhere. In particular, the shined value of $\chi_{L}$ on our brane is just given by the Yukawa potential in the transverse $n$ dimensions [19]:

$$
\langle\chi\rangle(x, y=0)=\Delta_{n}\left(\left|y_{*}\right|\right), \quad \Delta_{n}(r)=\left(-\nabla_{(n)}^{2}+m_{\chi_{L}}^{2}\right)^{-1}(r) .
$$

For $n>2$, 


$$
\begin{aligned}
\Delta_{n}(r) & \sim \frac{e^{-m r}}{r^{n-2}} \quad \text { for } m r \gg 1 \\
& \sim \frac{1}{r^{n-2}} \quad \text { for } m r \ll 1 .
\end{aligned}
$$

The resulting Majorana neutrino mass is suppressed by the factor $\Delta_{n}\left(\left|y_{*}\right|\right)$; restoring the dependence on $M_{*}$ we have

$$
m_{\nu}^{\text {Maj. }} \sim \frac{v^{2} \Delta_{n}(r)}{M_{*}^{n-1}} .
$$

This can give us an exponential suppression if $\chi_{L}$ is massive, while even if $\chi$ is very light, a power suppression is possible.

The case of massive $\chi_{L}$ can easily generate small enough Majorana masses, but is not particularly predictive without a theory specifying the inter-brane potential. On the other hand, if we consider very light $\chi_{L}$ [i.e. lighter than $1 / R$ but heavier than $\sim(\mathrm{mm})^{-1}$ to have escaped detection], and assume that the brane where $L$ is broken is as far away as possible, i.e. that $\left|y_{*}\right| \sim R$, then the neutrino mass is predicted to be

$$
m_{\nu}^{\text {Maj. }} \sim \frac{v^{2}}{M_{*}}\left(\frac{M_{*}}{M_{\mathrm{pl}}}\right)^{2-4 / n}
$$

where we have used $M_{*}^{n+2} R^{n} \sim M_{\mathrm{pl}}^{2}$. Note that for $n=4$, we recover the same rough prediction for neutrino masses as the old seesaw mechanism and the bulk right-handed neutrino. In this case there is a little more flexibility since the walls do not have to be quite so far away, and this can enhance the neutrino mass in the correct direction.

\section{NEUTRINO MASSES FROM THE BRANE-LATTICE CRYSTAL}

A qualitatively different possibility is raised if we are willing to contemplate a bulk populated with large numbers of branes. This possibility was raised in [16] in the context of stabilizing the extra dimensions; where the largeness of the extra dimensions was linked to the large brane number. For our purposes here we simply assume that the bulk is populated with a number density $n_{\text {brane }}$ of branes. In order to have a consistent picture of the brane lattice ignoring quantum gravitational effects, we must require that the lattice is dilute on the fundamental Planck scale: i.e.

$$
n_{\text {brane }} \ll M_{*}^{n} .
$$

Let us assume that the lepton number is broken on about half of the branes, while it is unbroken on the other half; our brane happens to be one where $L$ is unbroken. The information of $L$ breaking is transmitted to us by bulk messengers $\chi_{L}$ as in the previous section, leading to a neutrino mass

$$
m_{\nu}^{M a j} \sim \frac{v^{2}}{M_{*}^{n-1}} \int d^{n} y n_{\text {brane }} \Delta_{n}(|y|)
$$

Let us now suppose that $\chi_{L}$ is massive enough so that its Compton wavelength is smaller than the distance to the nearest wall. Then,

$$
\begin{aligned}
m_{\nu}^{\text {Maj. }} & \sim \frac{v^{2}}{M_{*}^{n-1}} \int d r r^{n-1} n_{\text {brane }} \frac{e^{-m_{\chi_{L}}{ }^{r}}}{r^{n-2}} \\
& \sim \frac{v^{2} n_{\text {brane }}}{M_{*}^{n-1} m_{\chi_{L}}^{2}} .
\end{aligned}
$$

It is perhaps most natural in this context to take $m_{\chi_{L}}$ $\sim M_{*}$, in which case the smallness of the neutrino mass is wholly controlled by the brane density. In the brane-lattice crystallization scenario for radius stabilization, this density was determined to be [16]

$$
\frac{n_{\text {brane }}}{M_{*}^{n}} \sim\left(\frac{M_{*}}{M_{\mathrm{pl}}}\right)^{4 / n} .
$$

Using this value for the density leads to a neutrino mass

$$
m_{\nu}^{\text {Maj. }} \sim \frac{v^{2}}{M_{*}}\left(\frac{M_{*}}{M_{\mathrm{pl}}}\right)^{4 / n} .
$$

Again the case $n=4$ leads to a neutrino mass of roughly the correct order of magnitude for solar and atmospheric neutrinos, with $n_{\text {brane }}$ and $m_{\chi_{L}}$ varying over reasonable ranges.

\section{PHENOMENOLOGICAL CONSTRAINTS}

The main constraints on any theory with SM fields localized on a 3-brane have to do with production of light bulk modes. The graviton is the one model-independent example of such a field, and graviton overproduction in astrophysical systems and in the early universe place unavoidable constraints on our framework, but do not exclude it [4]. As discussed in [19], if there are other light states in the bulk, such as vectors and scalars, even stronger bounds can result. The reason can be understood by simple dimensional analysis. The bulk graviton couples to dimension- 4 operators on the brane. As such, working in terms of the canonically normalized bulk graviton field $h_{A B}$, which has mass dimension ( $n$ $+2) / 2$, the coupling is schematically of the form

$$
\int d^{4} x \mathcal{O}_{4}(x) \frac{h}{M_{*}^{(n+2) / 2}}
$$

and therefore the cross sections for graviton emission scale with the energy as

$$
\sigma(\text { grav. prod. }) \sim \frac{E^{n}}{M_{*}^{n+2}} .
$$

By contrast, a vector field in the bulk couples to a dimension-3 operator on the wall, 


$$
\int d^{4} x \mathcal{O}_{3}(x) \frac{A}{M_{*}^{n / 2}}
$$

and the rate for bulk vector production is correspondingly enhanced

$$
\sigma(\text { vect. prod. }) \sim \frac{E^{n-2}}{M_{*}^{n}} .
$$

By this reasoning, the right-handed neutrino, coupling as it does to the lowest dimension SM invariant operator on our wall, should be most strongly coupled and potentially dangerous. However, it is important to remember that being a SM singlet, the bulk neutrino only interacts with SM fields via its mixing to $\nu_{L}$.

First consider putting the Higgs field to its VEV (we will return to processes involving physical Higgs fields at the end of this section). Then, the coupling of the right-handed neutrino to the wall neutrino generates a small Dirac mass as we have seen, with the lightest state being predominantly $\nu_{L}$ but having an admixture of the higher KK excitations of $\nu_{R n}^{c}$. For $n=2$, this mixing can be $O(1)$ and disastrous, while for higher $n$, even though the mixing to each state is small, the large multiplicity of states can still potentially give problems. It is most convenient to first go to the mass eigenstate basis. Then, the tower of $\nu_{R \vec{n}}^{c}$ KK states only interact through gauge interactions, with the vertices suppressed by $\theta_{\vec{n}}$ $\sim m_{\nu} /(|\vec{n}| / R)$. Let us consider the implications of this for early universe (but post "normalcy temperature" $T_{*}$ [4]) cosmology.

First, we have to determine whether any of these KK modes are ever thermalized. The worst case (biggest mixing angle) is for the first KK mode. The thermalization proceeds through through $W, Z$ exchange with ordinary SM particles, with a cross section

$$
\sigma \sim G_{F}^{2} T^{2} \theta^{2} .
$$

We determine the decoupling temperature as usual by equating $n \sigma v=H \sim T^{2} / M_{\mathrm{pl}}$, which yields

$$
T_{\text {dec. }} \sim 1 \mathrm{MeV} \theta^{-2 / 3} .
$$

For $n=2$, the situation is problematic, and likely too many of the heavy modes will be thermal during nucleosynthesis. However, already for $n=3$, the largest $\theta \sim 10^{-5}$ even taking $m_{v} \sim 3 \times 10^{-2} \mathrm{eV}$ for the atmospheric neutrino problem, and the decoupling temperature is forced above $\sim 1 \mathrm{GeV}$. Since in all cases, the normalcy temperature $T_{*} \lesssim 1 \mathrm{GeV}$, we can conclude that for $n>2$, the KK neutrinos are never thermalized once the universe becomes "normal." Of course, we have to ensure that they and, more importantly, bulk gravitons, are not created in thermal abundances before $T_{*}$, but that is a separate issue of the very early universe cosmology in this scenario which we will not address here.

Next, just like the non-thermalized bulk gravitons, there is the worry of evaporating too much energy into these bulk neutrino modes, unacceptably altering the expansion rate of the universe. First, we need to determine the rate at which any given KK mode of mass $m_{K K}$ decays back into SM states. The width is given by

$$
\begin{gathered}
\Gamma \sim G_{F}^{2} m_{K K}^{5}\left(\frac{m_{\nu}}{m_{K K}}\right)^{2} \\
\Gamma^{-1} \sim 10^{7} \mathrm{~s}\left(\frac{10^{-3} \mathrm{eV}^{2}}{m_{\nu}^{2}}\right)\left(\frac{1 \mathrm{GeV}}{m_{K K}}\right)^{3} .
\end{gathered}
$$

Note that the KK modes produced at temperatures beneath $\sim 1 \mathrm{GeV}$ are still around during nucleosynthesis. The rate which energy is evaporated into bulk neutrinos at temperature $T$ is

$$
\dot{\rho}_{\nu_{R}^{c}} \sim-\frac{T^{n+7} m_{\nu}^{2} G_{F}^{2} M_{\mathrm{pl}}^{2}}{M_{*}^{n+2}}
$$

to be compared with the normal cooling rate by adiabatic expansion

$$
\dot{\rho}_{\text {normal }} \sim-\frac{T^{6}}{M_{\mathrm{pl}}} .
$$

Requiring the normal rate to dominate over the neutrino rate at least for $T \sim \mathrm{MeV}$ when nucleosynthesis happens puts a rather mild bound on $M_{*}$,

$$
M_{*}>10^{(14-6 n) /(n+2)} \mathrm{TeV} .
$$

The reason for the weak bound is that production of bulk $\nu$ modes must proceed through a $W / Z$ and is therefore further suppressed by a $G_{F}^{2}$ factor. Of course we, in principle, have to worry about the decays of these bulk modes. The bulk gravitons which are produced have a long lifetime of order of the age of the universe and can unacceptably alter the background gamma ray spectrum when they decay. Bulk neutrinos are not as long lived and can be made to decay more harmlessly on a "fat brane" [4] just as in the case of bulk gravitons. Furthermore, if their decay to relativistic matter on the other brane, there is no worry that there decay products will ever overclose the universe. Other phenomenological constraints on right-handed neutrinos are similarly safe, for the same reasons.

One place for interesting signals could be in physical Higgs decays to $\nu_{L}+$ bulk neutrino. The width for the decay into any $\mathrm{KK}$ mode is suppressed by the neutrino Yukawa coupling $\lambda_{\nu}^{2}=m_{\nu}^{2} / v^{2}$, but there is an enhancement $\sim\left(m_{H} R\right)^{n}$ coming from the sum over all KK modes. The total decay width is

$$
\begin{aligned}
\Gamma_{H^{0} \rightarrow \nu_{L} \nu_{R}} \sim & \frac{m_{H}}{16 \pi} \times 10^{3-n} \times\left(\frac{m_{\nu}^{2}}{10^{-5} \mathrm{eV}^{2}}\right) \times\left(\frac{m_{H}}{100 \mathrm{GeV}}\right)^{n} \\
& \times\left(\frac{1 \mathrm{TeV}}{M_{*}}\right)^{n+2}
\end{aligned}
$$


This invisible decay for the Higgs field has a significant rate for $n=3$. A detailed analysis of novel Higgs physics, both in this scenario for generating neutrino masses as well as in extra-dimensional flavor theories will be reported elsewhere.

Finally, the constraints on light bulk $\chi_{L}$ messengers are essentially the same as those studied in [19], and just as the cases studied there, the exchange of the light $\chi$ field can give rise to attractive, isotope-dependent sub-millimeter forces $\sim 10^{6}$ times stronger than gravity, a signal that cannot be missed by the upcoming generation of sub-millimeter gravitational force experiments.

\section{LARGE NEUTRINO MAGNETIC MOMENTS}

As an example of other interesting neutrino physics in our scenario, we comment that it may be possible to generate large neutrino magnetic moments without neutrino masses. Suppose that there is an $S U(2)$ symmetry acting on the lefthanded doublets of the SM. Then, the $S U(2)$ invariant Majorana mass term $\nu_{a} \nu_{b} \epsilon^{a b}$ vanishes by antisymmetry. On the other hand, a magnetic moment operator of the form $\nu_{a} \sigma^{\mu \nu} F_{\mu \nu} \nu_{b} \epsilon^{a b}$ is not constrained to vanish. Note that this $S U$ (2) symmetry must be broken in order to generate charged lepton mass splittings. However, it is easy to arrange this while still forbidding neutrino masses. For instance, suppose that the flavor symmetry is $U(2)_{L} \times U(2)_{R}[19,20]$. If this symmetry is broken by a bi-fundamental, then charged lepton masses can arise, while Majorana neutrino masses are still forbidden. Since the UV cutoff in our framework is only $\sim \mathrm{TeV}$, we can have the magnetic moment operator suppressed by $\sim \mathrm{TeV}$, generating a large neutrino magnetic moment $\sim 10^{-19} \mathrm{e} \mathrm{cm}$ in the absence of a neutrino mass.

\section{CONCLUSIONS}

Theories that lower the fundamental scale of gravity close to $\mathrm{TeV}$ energies do not allow for the large desert in energy space between $\sim 10^{3}-10^{19} \mathrm{GeV}$ which have previously proven useful in model building. In particular, we seem to lose the seesaw mechanism for explaining small neutrino masses, since the requisite large energy scale for the righthanded neutrino mass is no longer at our disposal. In this paper we have shown that there are instead new, intrinsically higher-dimensional mechanisms for generating small neutrino masses. We explored two options. The first mechanism identifies right-handed neutrinos with any massless bulk fermions. These have volume suppressed couplings to the lefthanded neutrino localized on our three-brane, and can generate naturally small Dirac neutrino masses. The second mechanism takes advantage of the large infrared desert in our scenario: the large space in the extra dimensions. As an application of the general mechanism of [19], small Majorana neutrino masses can result if the lepton number is broken on distant branes, with the breaking being communicated to our wall by bulk messengers. In this paper we have been content to show that the neutrino mass scales required for explaining the atmospheric and solar neutrino problems can naturally arise in our framework, while we have left the flavor structure unspecified. Of course these could come about in a fairly standard way through flavor symmetries, although intrinsically higher-dimensional scenarios would be more interesting. We expect that in this and other areas, model building in extra dimensions will continue to be rich with fresh possibilities for phenomenology.

\section{ACKNOWLEDGMENTS}

It is a pleasure to thank G. Farrar, L.J. Hall, and A. Smirnov for valuable discussions. S.D. thanks the CERN theory group and J.M.R. thanks the Stanford University theory group for their respective hospitality during portions of this work. The work of N.A.H. was supported by the Department of Energy under contract DE-AC03-76SF00515. The work of S.D. was supported in part by NSF grant PHY9870115. The work of J.M.R. is supported in part by the A.P. Sloan Foundation.

\section{APPENDIX}

In this appendix we wish to show more explicitly that an interaction of the form of Eq. (A9), which is manifestly noninvariant under 5-dimensional Poincaré invariance, can nevertheless be generated in a theory where the 5D Poincare invariance is spontaneously broken by the domain wall on which $l, H$ are localized. Let $\Phi_{W}$ be a real scalar field whose VEV breaks some discrete $Z_{2}$ symmetry, the "kink" configuration interpolating between two vacua

$$
\left\langle\Phi_{W}(y \rightarrow \infty)\right\rangle=+\Phi_{\infty}, \quad\left\langle\Phi_{W}(y \rightarrow-\infty)\right\rangle=-\Phi_{\infty},
$$

gives rise to a domain wall. The position $y_{\text {wall }}$ of the wall in the fifth direction is arbitrary, so translations in this direction are spontaneously broken. The associated Nambu-Goldstone $g(x)$ just corresponds to the sound waves on the wall, which is to the deformations

$$
\Phi_{W}(x, y)=\left\langle\Phi_{W}[y+g(x)]\right\rangle .
$$

Following the same sorts of arguments as in [2], we can easily trap chiral fermions ( $l$ in this case) and scalars $(h)$ on the domain wall.

Let us recall how $l$ can be trapped. Introduce a 5-dimensional Dirac spinor

$$
L=\left(\begin{array}{c}
l \\
\bar{l}^{c}
\end{array}\right)
$$

which has a Yukawa coupling to the wall field

$$
\int d^{4} x d y \Phi_{W} \bar{L} L
$$

It is then well known that zero modes of the Dirac equation in the wall background exist of the form

$$
L=\left(\begin{array}{c}
f(y) l \\
0
\end{array}\right)
$$


where $f(y)$ is normalizable, i.e. $\int d y|f|^{2}=1$, whereas solutions of the form

$$
L=\left(\begin{array}{c}
0 \\
g(y) \bar{l}^{c}
\end{array}\right)
$$

are not normalizable $\int d y|g|^{2} \rightarrow \infty$. Therefore, $l$ but not $l^{c}$ is trapped to the wall. At distances large compared to the width of the wall, we can well approximate $f(y)=\sqrt{\delta(y)}$, and the localized zero mode is given by

$$
L(x, y)=\left(\begin{array}{c}
\sqrt{\delta(y)} l \\
0
\end{array}\right) .
$$

Notice that the dimensionalities match: $L$ is a $5 \mathrm{D}$ spinor of mass dimension 2, while $l$ is a 4D spinor of mass dimension $3 / 2$, the difference being made up by $\sqrt{\delta(y)}$ which has mass dimension $1 / 2$. Similarly, it is easy to trap scalar field $h$ on the wall from a bulk scalar field $H$ coupled to the wall field (for more details see [2]). Again, at long distances the localized mode is given as

$$
H(x, y)=\sqrt{\delta(y)} h(x) .
$$

Once again note that the mass dimensions match. Now, suppose that the theory also had the $\Psi$ Dirac fermion (not coupled to the wall field), which coupled to $H$ and $L$ via

$$
S^{\mathrm{int}}=\int d^{4} x d y \kappa H^{*}(x, y) \bar{\Psi}(x, y) L(x, y) .
$$

This gives some coupling between the trapped modes on the wall and $\Psi$, which can be read off by inserting Eqs. (A7), and (A8) into Eq. (A9)

$$
\begin{aligned}
S & =\int d^{4} x d y \kappa\left[\sqrt{\delta(y)} h^{*}(x)\right][\sqrt{\delta(y)} l(x)] \nu_{R}(x, y) \\
& =\int d^{4} x \kappa h^{*}(x) l(x) \nu_{R}(x, y=0)
\end{aligned}
$$

which is precisely the form of the interaction used in the main text.
[1] N. Arkani-Hamed, S. Dimopoulos, G. Dvali, and J. MarchRussell, presented by S.D. at SUSY 98, Oxford, 1998.

[2] N. Arkani-Hamed, S. Dimopoulos, and G. Dvali, Phys. Lett. B 429, 263 (1998).

[3] I. Antoniadis, N. Arkani-Hamed, S. Dimopoulos, and G. Dvali, Phys. Lett. B 436, 257 (1998).

[4] N. Arkani-Hamed, S. Dimopoulos, and G. Dvali, Phys. Rev. D 59, 086004 (1999).

[5] See, for example, J. Polchinski, "TASI lectures on D-branes," hep-th/9611050; C. Bachas, "Lectures on D-branes," hep-th/9806199.

[6] G.F. Giudice, R. Rattazzi, and J.D. Wells, Nucl. Phys. B44, 3 (1999); E. Mirabelli, M. Perlestein, and M. Peskin, Phys. Rev. Lett. 82, 2236 (1999); T. Han, J. Lykken, and R. Zhang, Phys. Rev. D 59, 105006 (1999); J.L. Hewett, Phys. Rev. Lett. 82, 4765 (1999).

[7] P. Argyres, S. Dimopoulos, and J. March-Russell, Phys. Lett. B 441, 96 (1998).

[8] K.R. Dienes, E. Dudas, and T. Gherghetta, Phys. Lett. B 436, 55 (1998); Nucl. Phys. B537, 47 (1999); K.R. Dienes, E. Dudas, T. Gherghetta, and A. Riotto, ibid. B543, 387 (1999).
[9] I. Antoniadis, Phys. Lett. B 246, 377 (1990).

[10] P. Horava and E. Witten, Nucl. Phys. B460, 506 (1996); E. Witten and J.D. Lykken, Phys. Rev. D 54, 3693 (1996); E. Caceres, V.S. Kaplunovsky, and I.M. Mandelberg, Nucl. Phys. B493, 73 (1997).

[11] G. Shiu and S.H.H. Tye, Phys. Rev. D 58, 106007 (1998).

[12] C. Bachas, J. High Energy Phys. 11, 023 (1998).

[13] R. Sundrum, Phys. Rev. D 59, 085009 (1999).

[14] R. Sundrum, Phys. Rev. D 59, 085010 (1999).

[15] V. Rubakov and M. Shaposhnikov, Phys. Lett. 125B, 136 (1983); G. Dvali and M. Shifman, Nucl. Phys. B504, 12 (1997).

[16] N. Arkani-Hamed, S. Dimopoulos, and J. March-Russell, Phys. Rev. D 63, 064020 (2001).

[17] C. Giunti, C.W. Kim, and M. Monteno, Nucl. Phys. B521, 3 (1998).

[18] See e.g. J.N. Bahcall and P.I. Krastev, Phys. Rev. D 53, 4211 (1996).

[19] N. Arkani-Hamed and S. Dimopoulos, hep-ph/9811353.

[20] Z. Berezhiani and G. Dvali, Phys. Lett. B 450, 24 (1999). 